\title{
Configuración de aspiraciones educativas: un análisis comparado de las subjetividades y narrativas de los jóvenes chilenos*
}

Configuration of Educational Aspirations:

a Comparative Analysis of the

Subjectivities and Narratives

of Young Chileans

Configuração de aspirações educacionais: uma análise comparativa das subjetividades e narrativas de jovens chilenos

Maria Jose Valdebenito** (iD orcid.org/0000-0002-9231-188X

Para citar este artículo: Valdebenito, M. J. (2020). Configuración de aspiraciones educativas: un análisis comparado de las subjetividades y narrativas de los jóvenes chilenos. Revista Colombiana de Educación, /(80), 101-120. https://doi.org/10.17227/rce.num80-9456

\section{(c) $\underset{\mathrm{BY}}{(\mathrm{NC}}$}

Recibido: 19/12/2018

Evaluado: 10/09/2019

* Este artículo se ha desarrollado en el marco del estudio Liderando proyectos juveniles de Cedle.

** Candidata a Doctora en Ciencias Sociales de la Universidad de Buenos Aires. Directora Magíster en Política Educativa en la Académica Facultad de Educación, Universidad Alberto Hurtado, Chile. Correo electrónico: mavaldeb@uahurtado.cl 


\begin{abstract}
Resumen
Este artículo presenta los resultados de una investigación de carácter cualitativa que tuvo como propósito analizar los procesos de decisión vocacional de dos grupos de jóvenes de último año de la enseñanza secundaria en Chile, uno de ellos perteneciente a la modalidad de estudios científico-humanística y el otro a la modalidad técnico-profesional. Se profundiza en las subjetividades implicadas en la definición de rutas educativas y laborales futuras, sus expectativas, aspiraciones y los soportes institucionales dispuestos. El estudio se inscribe en el campo de las investigaciones de las transiciones educativas y orientación vocacional, bajo la mirada de las subjetividades y narrativas juveniles. A partir de tres ejes analíticos: identidad, posición socioestructural y voz, se reconocen las narrativas y experiencias presentes en la definición de los proyectos posteriores al egreso. Como principal hallazgo se observa que los jóvenes de la educación técnico-profesional, a diferencia de los de la modalidad científico-humanista, proyectan recorridos de mayor complejidad futura que les exige asumir una posición activa frente a los desafíos y barreras que deben sortear. La combinación de estudios y trabajo, así como la configuración de identidades profesionales anticipadas, constituyen elementos relevantes en las experiencias. Por su parte, los soportes institucionales provistos por los establecimientos educativos de la educación media técnico-profesional representan las principales barreras para los jóvenes.
\end{abstract}

\section{Palabras clave}

aspiración; expectativa decisiones vocacionales; orientación vocacional; transiciones educativas

\section{Keywords}

aspiration; expectation; vocational decisions; vocational orientation; educational transitions

\begin{abstract}
This article presents the results of a qualitative research that aimed to analyze the vocational decision making processes of two groups of students in their last year of secondary education in Chile. One of the groups follows the scientific-humanistic studies modality, while the other follows the technical-professional modality. The study examines the subjectivities implied in the definition of the future educational and work paths, their expectations, aspirations, and the institutional support in place. The study is part of the field of research on educational transitions and vocational orientation, under the gaze of youth subjectivities and narratives. Starting from three analytical core concepts: identity, socio-structural position and voice, the authors identified the different stories and experiences involved in the definition of the students' projects after completing their education. One of the main findings is that the youths in the technical-professional modality project more complex future trajectories than their academically oriented counterparts, where the former must have an active role to deal with the barriers and challenges they will face. Combining work and study as well as the configuration of early professional identities are important elements in these experiences. On the other hand, the institutional support provided by the educational establishments of technical-professional secondary education represent the main barriers for the youths.
\end{abstract}

\section{Resumo}

Este artigo apresenta os resultados de uma pesquisa qualitativa cujo objetivo foi analisar os processos de decisão vocacional de dois grupos de jovens do último ano do ensino médio no Chile, um deles pertencente à modalidade de estudos científico-humanísticos e a outro à modalidade técnico-profissional. Investiga as subjetividades envolvidas na definição de futuros rumos educacionais e de trabalho, suas expectativas, aspirações e os suportes institucionais disponíveis. O estudo insere-se no campo de investigação das transições educacionais e da orientação profissional, sob o olhar das subjetividades e narrativas juvenis. A partir de três eixos analíticos: identidade, posição socioestrutural e voz, são reconhecidas as narrativas e experiências presentes na definição dos projetos de pós-graduação. Como principal achado, observa-se que os jovens na formação técnico-profissional, diferentemente dos da modalidade científico-humanista, projetam roteiros de maior complexidade futura que os obrigam a assumir uma posição ativa diante dos desafios e barreiras que devem superar. A combinação de estudos e trabalho, bem como a configuração de identidades profissionais antecipadas, constituem elementos relevantes nas experiências. Por outro lado, os apoios institucionais proporcionados pelos estabelecimentos de ensino secundário técnico-profissional representam as principais barreiras para os jovens.

\section{Palavras-chave}

aspirações; expectativas; decisões vocacionais; orientação vocacional; transições educacionais 


\section{Introducción}

Transformaciones en distintas esferas del entramado social, como el debilitamiento de las instituciones, las nuevas orientaciones normativas, los ajustes en los mercados laborales y la naturaleza del trabajo han cambiado el escenario de los recorridos y los destinos educativos y laborales de los jóvenes. En este marco, es posible observar un creciente interés investigativo por las aspiraciones y los procesos de adecuación de expectativas a las características y oportunidades del entorno de los jóvenes (Elder y Giele, 2009; Heinz y Kruger, 2001). La valoración de este tipo de estudios remite no solo a las posibilidades de evaluar el grado de satisfacción o cumplimiento de metas individuales, sino que también provee un marco de referencia que permite configurar un marcador social que da cuenta de los cambios que experimentan las sociedades y que expresan, de manera singular, a las distintas generaciones.

En el caso chileno, observamos importantes y aceleradas mutaciones socioculturales e institucionales experimentadas por su sociedad, que vuelven relevante el analisis de las subjetividades y nuevas orientaciones juveniles. En las últimas dos décadas, el país ha tenido un importante progreso en las tasas de finalización de educación secundaria, que han estado acompañadas de un aumento exponencial de las aspiraciones de acceso a la educación superior por parte de los jóvenes y sus familias (CIDE, 2012). Las cifras en torno al ingreso efectivo a las instituciones de educación superior se han triplicado desde la recuperación de la democracia en 1990; se observan tasas de ingreso al sistema de educación superior por cada cohorte superiores al $60 \%$ del total de egresados de la enseñanza secundaria. El crecimiento de estos indicadores se ha mantenido constante, particularmente en los sectores de menores ingresos que, hasta hace poco, evidenciaban un rezago significativo con relación al resto.

No obstante las alentadoras cifras en términos de cobertura, el acceso no ha ido acompañado de condiciones de equidad. En primer lugar, se evidencian significativos niveles de desigualdad de acceso a la educación superior de la población escolar. El sistema de educación superior muestra una alta segregación socioeconómica que se ve representada en la existencia de instituciones y carreras de menor prestigio académico y selectividad a la que asisten jóvenes provenientes de sectores medios y bajos (Programa de las Naciones Unidas para el Desarrollo-PNUD, 2017; Torres y Zenteno, 2011). Esta inequidad es un reflejo de la segmentación del sistema escolar y las desigualdades del país, que se profundizan por su modelo institucional. En el caso de la educación secundaria, el sistema se divide en dos vertientes formativas: una educación científica o humanista (ЕмнС), de la que egresa el $62 \%$ de los estudiantes, y una técnica (ЕмтP), de la que egresa el $38 \%$. Si bien el modelo enfocado a la formación

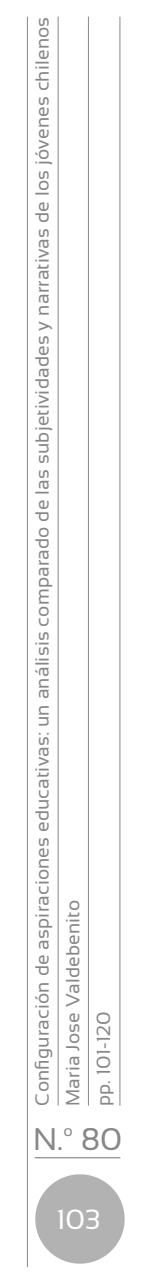


técnica está orientado a la inserción laboral temprana, en los últimos años se observa un incremento de la participación de sus egresados de la EMTP en la educación superior. Esta inserción de los jóvenes de la educación técnica en la educación superior ha demostrado ser compleja por diversos factores, en especial por sus diferenciales de capitales económicos, culturales y los énfasis de la formación recibida. Estudios como los de Larrañaga et al. (2013) y Sepúlveda y Valdebenito (2018) han evidenciado que los estudiantes de la EMTP presentan trayectorias complejas, de alta propensión a las discontinuidades, en comparación con los de la EMHC.

Las ideas expuestas hasta acá exigen no solo analizar las trayectorias posteriores al egreso, sino profundizar en interrogantes relativas a las subjetividades implicadas en sus procesos de decisión vocacional. Conocer el valor y significado que le otorgan los jóvenes a la educación superior, el peso asignado a esta experiencia en sus trayectorias y proyectos futuros, asi como la delimitación de rutas y recorridos posteriores al egreso son cuestiones fundamentales por atender. A través del análisis de las expectativas, las aspiraciones, los marcos de orientación y las perspectivas de realización y concreción de estas para jóvenes de distintos contextos, nos situaremos no solo frente a interrogantes acerca del sentido de agencia, control y estructuras, sino también en los procesos de individualización experimentados por los jóvenes y la sociedad chilena en su conjunto.

\section{Marco teórico}

En el campo de las ciencias sociales, el análisis de las aspiraciones y expectativas juveniles ha enfatizado en el efecto de las políticas destinadas a aumentar la legitimidad de determinadas trayectorias educativas y laborales, formas de empleo y proyectos de vida, en mayores oportunidades de acceso a espacios que han estado clausurados para gran parte de la población (Furlong y Cartmel, 1997, Gibbons y Vignoles, 2009, Hart, 2013) y en la democratización e igualdad social a partir la masificación de la educación superior (Grytnes, 2011; Lehmann, 2004).

En este marco, los estudios sobre la configuración de las aspiraciones y expectativas han sido un ámbito emergente pero creciente, que a través de su profundización ha permitido ahondar en los interrogantes acerca de la dialéctica entre estructura y agencia implicados en los procesos de construcción de proyectos (Ball et al., 2002; Sellar y Gale, 2011).

El debate emerge como una forma de contrarrestar la mirada tradicional de los procesos de definición vocacional, que los ha concebido como procesos simples, técnicamente racionales, donde los jóvenes evalúan sus propias habilidades, intereses y oportunidades disponibles. Como lo expresan Bennett et al. (1992): "suponemos que, conociendo sus capacidades y 
otras características personales, los individuos realizan una estimación de los ingresos esperados de lo que les reportará la educación, formación y el mercado de trabajo" (citados en Hodkinson y Sparkes, 2006, p. 31).

Esta perspectiva se ancla en la denominada teoría de los rasgos, que enfatiza en un fuerte sentido de individualidad y racionalidad, perdiendo por completo de vista los constreñimientos estructurales en que se desenvuelven las experiencias de vida de los jóvenes.

En un intento por superar esta perspectiva, desde la sociología de la educación los estudios han argumentado que las decisiones no son determinadas por el individuo sino que más bien se ven afectadas por estructuras que configuran las concepciones de lo que es (in)deseable, posible y factible (Roberts, 1968, 1975). Así, existiría un conjunto de normas, reglas, instituciones, convenciones prácticas y discursos que restringen o habilitan a un grupo determinado de actores para la ejecución de ciertas acciones (Dale et al., 2016), siendo el mercado laboral juvenil, la organización de producción, el empleo, la regulación gubernamental y la clase social las estructuras de oportunidad que pueden incidir con mayor peso en la toma de decisiones.

En este enfoque, Hodkinson (2006) ha demostrado que las decisiones son tanto pragmáticas como racionales y que existe un nexo intrínseco o equilibrio entre lo social y lo racional. Los aspectos no racionales se resocializan y los agentes toman decisiones con base en la eficacia de los logros deseables, y evalúan estos últimos a la luz de su marco de valores personales más que de la utilidad personal (Hatcher, 1998). En consecuencia, tanto la agencia individual como las estructuras ocupacionales y sociales asumen un rol activo y no forman parte solo del contexto externo. Se comprende que las decisiones son pragmáticas, están relacionadas con el contexto y vinculadas inexorablemente a los antecedentes familiares, la cultura y las historias de vida de los jóvenes; están basadas, podemos decir, en contactos sociales y experiencias vitales, y se instauran al mismo tiempo como racionales e irracionales (Hodkinson y Sparkes, 2006). Son parcialmente racionales, siendo motivadas por sentimientos y emociones, la adopción de una opción más que la elección de una entre muchas (Ball et al., 2000).

Los individuos toman decisiones dentro de horizontes para la acción, siendo el habitus y las estructuras de oportunidad los factores influyentes en la percepción de las opciones o alternativas que pudieran estar disponibles. En este sentido, las oportunidades no solo están a disposición para ser elegidas, pueden crearse por las presiones locales y el ethos derivados de la propia historia de los jóvenes y que generan un impacto significativo en la determinación de lo que es posible para ellos (Atkins, 2017). Como señalan Ball et al. (2000), estos horizontes son percepciones sociales, espaciales y temporales asociadas a costos, confianza, preocupación, expectativas, tradición y comunidad. Así, al profundizar en los conocimientos, valores

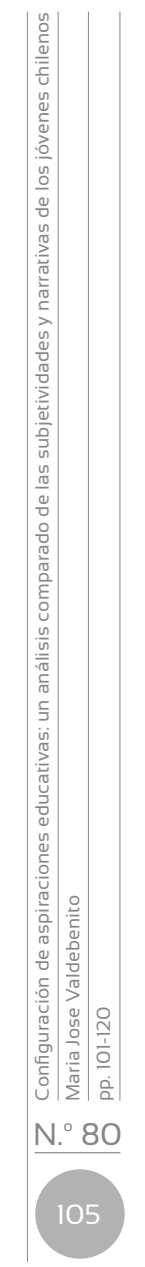


y normas de acción con que las personas crecen y evolucionan, Ilegamos a comprender cómo internalizamos nuestros "mapas de significados" (Clarke et al., 1982), que expresan cómo se experimentan, comprenden e interpretan dichas relaciones. En este sentido, se vuelve manifiesta la necesidad de que las decisiones sean leídas en términos de historia de vida, como un proceso profundo y complejo en el que la identidad del sujeto evoluciona a partir de la interacción social y la cultura en que se ha desenvuelto su vida.

En este marco, el análisis del rol de las familias y las escuelas es fundamental (Cabrera, y La Nasa, 2002; Jacinto, 2010; Rivera, 2012). La escuela, en particular en su rol de socialización, aporta información y las herramientas que influencian la toma de decisiones, lo que permite incidir, refinar o modificar el habitus y el horizonte de acción (Hodkinson y Sparkes, 2006). Las instituciones escolares pueden ayudar a la apropiación, al refuerzo y a evitar el desarrollo de distintas lógicas sociales, lo que configura una relación de dependencia recíproca entre individuos y procesos sociales (Elias, 1997).

En este escenario de configuración, las instituciones educativas en su operación podrían imponer ciertos desafíos en los propios procesos de construcción identitaria y subjetividad, que ellas mismas podrían cumplir; esto, como señala Dubet (2011), da lugar a un "acto de delegación de responsabilidad a los individuos". Desde esta perspectiva, las instituciones escolares - $\mathrm{O}$, más bien, las pruebas a las que se someten los estudiantes— podrían llegar a alimentar la existencia de un sistema que invalida o invisibiliza el peso de las posiciones sociales y los diferenciales de oportunidad que se asocian con ellas, lo que desencadena experiencias dolorosas y solitarias cuando se asumen como faltas o errores. En el caso chileno, autores como Araujo y Martuccelli (2012) han advertido que las estrategias posicionales desplegadas obligan a los actores a lidiar constantemente con las inconsistencias transversales de las posiciones sociales.

En este sentido, se alude a que la nueva cultura escolar no solo contribuiría a reproducir aún más los mecanismos discriminatorios en la trayectoria escolar de los alumnos, sino también a reproducir desigualdad al despertar confianza en algunos estudiantes, y en otros, inseguridad. En los procesos de orientación vocacional se transmite a los jóvenes que son responsables de su fracaso escolar; esto origina una renuncia y una interiorización de las oportunidades objetivas de éxito (Araujo y Martucelli, 2012; Dubet, 2005). En este marco, la selectividad académica y social instaurada en las instituciones de educación opera como una experiencia de inclusión-exclusión: mientras que los "buenos" estudiantes o la "élite" son acogidos por el sistema y ven alzarse frente a ellos una integración escolar, "la masa de los estudiantes es dejada a su suerte, sometida a las angustias del fracaso y al miedo a la exclusión" (Dubet, 2005, p. 68). 


\section{Metodología}

La investigación se planteó un enfoque metodológico que profundiza en la mirada a las subjetividades y narrativas de los jóvenes sobre la configuración de sus aspiraciones y expectativas educativas y los soportes existentes en ese proceso. Siguiendo una estrategia de indagación cualitativa se enfatizó en un levantamiento inductivo a partir de la propia experiencia de los sujetos. Con el fin de profundizar en el proceso de construcción de proyectos educativos posteriores al egreso de los jóvenes, se desarrollaron entrevistas individuales que trataron de comprender los sentidos implicados en las decisiones vocacionales y delimitación de estrategias y rutas de dos grupos de estudiantes: los de la educación media humanista-científica (ЕмHC) y los de la educación técnica (ЕмтP). Metodológicamente se trabajó con un enfoque narrativo de relatos de experiencias biográficas y se profundizó en los denominados puntos de quiebre (Hodkison y Sparkes, 1997) y momentos críticos implicados en las experiencias escolares. De esa manera se exploró lo que los jóvenes hacen o quieren hacer en aquellos momentos de toma de decisiones. A partir del relato de los sujetos acerca de sus procesos de toma de decisiones y experiencia escolar se intentó identificar las lógicas narrativas, o posiciones con base en las cuales los sujetos estructuran y cuentan sus decisiones y experiencias. El valor de este enfoque radica en que a través del reflejo de experiencias pasadas de los sujetos se incluyen narraciones densas en valores, creencias y actitudes con alto trasfondo social (Bertaux, 1982). En este sentido, las narrativas no solo facilitan la comprensión de los procesos biográficos de un individuo, sino que también permiten dar cuenta de biografías colectivas. Tomando las reflexiones de Arfuch (2007), esta perspectiva analítica se caracteriza por una puesta de sentido de la vida del sujeto, en la cual el otro, es decir el entrevistador o entrevistadora, es quien aparece en la escena como detonador del conocimiento de uno mismo. Así, las narrativas biográficas se comprenden como productos de prácticas y experiencias situadas social e históricamente.

Considerando este enfoque, las técnicas de abordaje y de trabajo buscaron cubrir diversidad de experiencias, en función de distintos atributos como modalidad de estudios, género y desempeño escolar. Para ello se realizó una selección intencionada de dos grupos de estudiantes asociados a seis establecimientos educativos de niveles socioeconómicos similares (NSE medio y medio bajo), tres de la educación secundaria científico-humanista (EMCH) y tres de educación media técnico profesional (EMTP). 
Tabla 1.

Matriz de selección de estudiantes

\begin{tabular}{ll}
\hline \multirow{2}{*}{ Género } & \multicolumn{1}{c}{6 hombres } \\
\cline { 2 - 2 } Modalidad de estudios & 6 de EMTP \\
\cline { 2 - 2 } & 6 de HC \\
\hline \multirow{2}{*}{ Desempeño } & 6 de desempeño superior a la media de curso \\
\hline
\end{tabular}

Fuente: elaboración propia.

El análisis se llevó a cabo en distintas etapas. En un primer momento, se configuraron los relatos y luego se inició un proceso de análisis comparado de las narrativas y de las experiencias emergentes en estas. Este trabajo, a través de un procedimiento de comparaciones constantes, permitió dar consistencia a los conceptos y favorecer su transversalidad.

El modelo analítico estuvo compuesto por tres ejes definidos en una fase preliminar. El primero, asociado a los procesos de construcción identitaria, que permitió examinar las subjetividades asociadas a la configuración de aspiraciones y expectativas, incluyendo sus atribuciones respecto de su experiencia previa al egreso, su capacidad de agencia y las disposiciones hacia el trabajo y estudios. El segundo, de posiciones socioestructurales, permite analizar la vinculación de las aspiraciones posteriores al egreso con pruebas y soportes estructurales, que marcan sustantivamente los recorridos de los sujetos, y en ese sentido expresan la trama de correspondencia entre individuo y sociedad. El tercero, asociado a la voz emergente en las experiencias de transición.

\section{Tabla 2.}

Ejes analíticos

\begin{tabular}{|c|c|}
\hline Ejes & Contenidos \\
\hline \multicolumn{2}{|l|}{ Construcción identitaria } \\
\hline \multirow{4}{*}{$\begin{array}{l}\text { Eje que identifica las lógicas } \\
\text { narrativas o posiciones a partir de } \\
\text { las cuales los sujetos estructuran } \\
\text { y cuentan su trayectoria. Reflejan } \\
\text { las formas de construcción } \\
\text { identitaria de los jóvenes. En cada } \\
\text { lógica, el sujeto se muestra al otro } \\
\text { (en este caso al entrevistador), } \\
\text { diferenciándose de los demás, } \\
\text { así revela formas de construcción } \\
\text { identitaria (Gaete y Soto, 2012,). }\end{array}$} & $\begin{array}{l}\text { 1. Las lógicas de diferenciación individual: } \\
\text { esfuerzos del individuo por resaltar su } \\
\text { singularidad y diferencias respecto de sus pares. }\end{array}$ \\
\hline & $\begin{array}{l}\text { 2. Nivel de agencia declarado: atribuciones } \\
\text { causales sobre el propio rol en la trayectoria } \\
\text { y percepciones de autoeficacia. }\end{array}$ \\
\hline & $\begin{array}{l}\text { 3. El sentido atribuido al cambio y a la } \\
\text { movilidad: aspiraciones de movilidad } \\
\text { o estabilidad proyectada. }\end{array}$ \\
\hline & $\begin{array}{l}\text { 4. Las estructuras temporales: focos temporales } \\
\text { desde donde se estructuran las narrativas. }\end{array}$ \\
\hline
\end{tabular}




\begin{tabular}{|c|c|}
\hline Ejes & Contenidos \\
\hline \multicolumn{2}{|l|}{ Posiciones sociohistóricas } \\
\hline \multirow{4}{*}{$\begin{array}{l}\text { Eje que identifica los soportes } \\
\text { institucionales y su vinculación } \\
\text { con el lugar ocupado en la } \\
\text { estructura social, relevando } \\
\text { sus percepciones y eventuales } \\
\text { efectos en los recorridos }\end{array}$} & $\begin{array}{l}\text { 1. Soportes familiares: rol atribuido a la familia } \\
\text { en el ingreso, la secuencia y los cambios } \\
\text { en la trayectoria educativa laboral. }\end{array}$ \\
\hline & $\begin{array}{l}\text { 2. Soportes escolares (institucionales): } \\
\text { rol atribuido a la escuela y su modelo } \\
\text { formativo en el ingreso, la secuencia y } \\
\text { los cambios en la trayectoria educativa } \\
\text { laboral. Instancias de apoyo vocacional. }\end{array}$ \\
\hline & $\begin{array}{l}\text { 3. Adscripción significativa: percepción } \\
\text { de pertenencia a clase social vinculada } \\
\text { a las trayectorias futuras. }\end{array}$ \\
\hline & $\begin{array}{l}\text { 4. Percepción de la estructura de } \\
\text { oportunidades y su asociación con la } \\
\text { trayectoria posterior al egreso. }\end{array}$ \\
\hline \multicolumn{2}{|l|}{ Voz } \\
\hline \multirow{4}{*}{$\begin{array}{l}\text { Eje que releva la continuidad } \\
\text { o desintegración con base } \\
\text { en los contenidos y vivencias } \\
\text { subjetivas asociadas al o } \\
\text { los hitos de transición. }\end{array}$} & $\begin{array}{l}\text { 1. Integración de vivencias: continuidad } \\
\text { o desintegración con base en los } \\
\text { contenidos y vivencias subjetivas } \\
\text { asociadas al o los hitos de transición. }\end{array}$ \\
\hline & $\begin{array}{l}\text { 2. Conexión de aspiraciones, expectativas y } \\
\text { acciones en los relatos transicionales. }\end{array}$ \\
\hline & $\begin{array}{l}\text { 3. Esfuerzo: Intensidad del esfuerzo percibido. } \\
\text { Espacios de tensión y desgaste asociados a los } \\
\text { procesos de transición. (esfuerzo-meritocracia). }\end{array}$ \\
\hline & $\begin{array}{l}\text { 4. Acceso a soportes institucionales } \\
\text { para la toma de decisiones. }\end{array}$ \\
\hline
\end{tabular}

Fuente: elaboración propia.

Los resultados han sido analizados a la luz de los tres ejes analíticos expuestos y su presentación se articula en torno a ellos. A continuación se desarrollan los principales hallazgos de cada eje y se presenta una gráfica que sintetiza la fuerza con la que se presentan las dimensiones implicadas en cada grupo. La gráfica da cuenta de la intensidad narrativa que se identifica en los relatos, la cual, al mostrarse de manera agregada para los grupos EMHC Y EMTP, ayuda a apreciar las diferencias con mayor claridad.

\section{Resultados}

\section{Eje identitario}

En una mirada general a los relatos, los discursos dan cuenta de que el sistema de educación superior ha dejado de ser una instancia destinada exclusivamente para las élites. Aunque se observan diferencias entre los jóvenes, sus narrativas ponen de manifiesto la influencia que han tenido en 
Chile las políticas orientadas a promover la adquisición de las credenciales educativas. En general, los discursos expresan la extensión del sistema de educación superior a todos los sectores sociales y de la apreciación de los estudios superiores como un medio importante para asegurar cierto nivel de bienestar o prosperidad futura.

Las ambiciones juveniles no solo se anclan en la idea de que la educación superior es un mecanismo de movilidad social, sino que esta se concibe como la posibilidad para configurar un proyecto que conjuga el desarrollo profesional con los intereses personales. En este sentido, el valor de la educación superior tiene un sentido instrumental y vocacional.

No obstante lo anterior, la educación superior adquiere distintos valores según los intereses. Por ejemplo, para muchos jóvenes, en especial los de la EMTP, se configura una orientación con mayor énfasis instrumental, en la que la formación postsecundaria constituye un medio para alcanzar y garantizar inserción y estabilidad laboral. Para otros, mayoritariamente los jóvenes próximos a egresar de la EMHC, tiene un mayor valor vocacional, ya que brinda posibilidades tanto de movilidad social como de desarrollo personal. Es una instancia que permite profundizar en ámbitos específicos de interés individual, facilitando la configuración de un proyecto en el que el quehacer profesional se asocia a un sentido social o de desarrollo individual. Dicho lo anterior, es necesario precisar que en ningún caso se puede afirmar que los posicionamientos están disociados, sino que en muchos casos se integran discursivamente.

Mi meta más cercana es hacer la carrera de administración de empresas a la vez que trabajo. Me gustaría estudiar en el DuOc, en concreto en el centro que está ubicado en Alonso Ovalle porque queda muy cerca de mi trabajo y facilitaría la compatibilización de los estudios con el trabajo. (Mujer, EMTP)

Si no cumplo mi sueño sería terminar como cualquier típico oficinista que tiene un horario de la mañana a la noche, que está todo estresado porque realmente no está haciendo lo que quiere. Eso lo encuentro terrible. (Hombre, EMHC)

En consonancia con las potencialidades de desarrollo personal y profesional asociados al paso por la educación superior, los jóvenes consideran este proceso como una etapa preparatoria hacia la adultez. La educación superior para los jóvenes es una etapa preparatoria que configura la matriz de transición hacia la adultez — definida por los propios jóvenes como una etapa de la vida a la que se transita cuando se experimenta independencia y autonomía-. Como había constatado Dubet (2005), hoy las elecciones de carreras tienden a estar orientadas a prolongar la condición de estudiantes y a construir una juventud fundamentada en una autonomía progresiva. 
Frente a este hallazgo, emerge una diferenciación en las narrativas de los jóvenes. Para los jóvenes de EMHC, el paso por la educación superior se concibe como una compleja etapa de realización individual y preparatoria al mundo laboral. La prosperidad futura asociada al paso por la vida en la educación superior eclipsa, por así decir, la proyección de desarrollo de actividades de búsqueda y/o tanteo vocacional en las que las inserciones laborales tempranas constituyen instancias significativas de construcción identitaria. En las narrativas de los jóvenes de la EMTP, que mayormente desean alternar estudios y trabajo, predomina la idea de que el trabajo aporta herramientas de aprendizaje para manejar la vida y da sustento a los procesos de autonomía y consolidación personal. En este periodo los jóvenes atribuyen un significativo valor a la realización personal a través del trabajo y a las eventuales posibilidades de progreso en el ámbito laboral, además de las posibilidades de autonomía económica. El ingreso al mundo laboral temprano, podemos afirmar, constituye para estos jóvenes de la EMTP una contribución significativa en su tránsito a la adultez. En consonancia con lo señalado, las proyecciones futuras vinculadas a la adultez y el valor otorgado a la independencia también muestran aspectos diferenciados entre las narrativas: para los jóvenes de la EMTP, es decir, de ingreso más rápido al mundo del trabajo, el valor otorgado a la independencia y la significación a la movilidad social adquieren mayor preponderancia.

[nunca se ha barajado]... lo de trabajar mientras esté estudiando, pues tanto mis papás como yo creemos que lo mejor ahora es que me enfoque completamente en estudiar. (Mujer, EMHC)

De hecho, creo que ser adulto tiene que ver un poco con eso. Con estar trabajando en lo que a uno le gusta, poder tener su plata y disponer de uno, que es un poco lo contrario a lo que ocurre cuando uno es joven, que tiene que andar pidiendo permiso y plata para salir, esas cosas. Lo que pasa a veces cuando uno es joven, es que se cree grande, pero en realidad no lo es. Ahí a veces, se genera mucha irresponsabilidad. (Hombres, EMTP)

No obstante las diferencias, frente al futuro, la mayoría de los jóvenes se auto representa como personas capaces de hacer frente al contexto de oportunidades y a los desafíos proyectados. Esta actitud creativa no está necesariamente vinculada a la asunción de una posición activa en estos procesos sino a la auto-eficacia, es decir, al auto reconocimiento de capacidades y habilidades potenciales que los habilitaría a hacer frente a las dificultades de futuro.

Eso en definitiva es lo único que podría imposibilitar que no pueda seguir estudiando, porque tengo todo el apoyo de mis más cercanos, soy muy perseverante, metódica, y planificada. Lo que me propongo, lo cumplo, aunque me demore. (Mujer, EMHC) 
Considero que uno es el exclusivo responsable de las cosas que le pasan. En esa línea, creo que las cosas que van a pasarme dependen de mí y de nadie más, aunque hay que reconocer que en la vida hay cosas que se programan, y otras que simplemente suceden. Así, creo que lo único que podría atentar contra los proyectos que tengo es el estrés laboral, pero todavía hay que ver qué pasa más adelante con eso. (Hombres, EMTP)

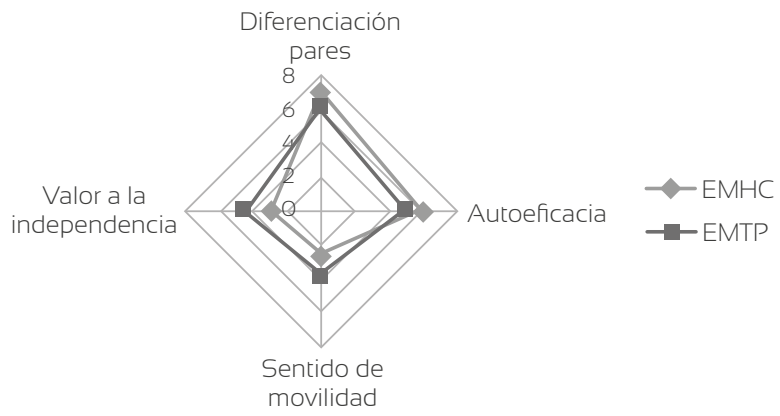

Figura 1. Dimensiones identitarias según modalidad de estudios Fuente: elaboración propia.

\section{Eje posicional socio estructural}

El paso por la educación superior constituye para la mayoría de los jóvenes chilenos un evento personal que es, a su vez, transgeneracional. La participación en la educación superior se abre como una posibilidad, como un anhelo familiar y, en algunos casos, como un deber para las generaciones "jóvenes". Las familias reconocen la importancia de la prosecución de estudios y ven ella la posibilidad de que sus hijos participen de espacios de desarrollo e inclusión social. Los jóvenes procedentes de familias de bajos capitales, como señala Canales et al. (2016), asumen el acceso a los estudios terciarios como un encargo familiar o, en otras palabras, como una interpelación de sus familias a participar en espacios académicos que habían permanecido clausurados socialmente para sus padres.

A pesar del peso implícito, los jóvenes no perciben las expectativas y aspiraciones familiares como nudos de tensión, sino más bien como un impulso para cumplir las metas. Si bien estas orientaciones familiares inicialmente se conciben como prescriptivas, pierden este carácter cuando los estudiantes reflexionan sobre el proceso de toma de decisión final. Los jóvenes en sus relatos no expresan sentirse oprimidos, sino motivados a proseguir con los estudios superiores, declarándose por sobre todo autónomos en la toma de decisiones. Los jóvenes que desean continuar sus estudios no cuestionan la decisión tomada y el rol que han podido ejercer sus familias en la elección de sus recorridos posteriores al egreso. 
Así, la decisión de qué estudiar en definitiva la tomé yo solo. Averigüé un poco por aquí y por allá, pero a la larga, nadie influyó en mi decisión. Claro, hubo algunos factores que incidieron (como por ejemplo, lo que costaba odontología, que era algo que no iba a poder pagar aunque tuviera crédito), pero al final la decisión pasó por mí mismo y por nadie más. (Hombre, HC)

La carga que puedan sentir los estudiantes de continuar sus estudios por cumplir con las expectativas familiares se manifiesta con mayor fuerza entre aquellos jóvenes EMTP que no proyectan un ingreso inmediato a la educación superior, quienes en algún sentido dan cuenta de su deuda. No obstante lo anterior, este sentido de deuda no se expresa como dramática, por el contrario, se sigue manifestando optimismo y esperanza al respecto.

En mi casa están contentos con el hecho de que esté trabajando ya, pero igual me dicen de cuando en cuando que sería bueno que estudiara algo más. Por mi parte, debo decir que igual me gustaría estudiar, pero por otro lado siento que estoy bien así. (Mujer, EMTP)

Al profundizar en los relatos es posible reconocer un arreglo familiar basado en el apoyo y soporte familiar que genera las bases para la prosecución de estudios. Los jóvenes de sectores Hc valoran las estrategias desarrolladas por sus familias para ayudarlos a alcanzar alternativas ajustadas a sus intereses y expectativas, por ejemplo tratar de beneficiarse de medios de financiación para los estudios brindados por las instituciones como créditos y becas. Estos gestos de parte de la familia, lejos de generar un sentimiento de miedo al fracaso, se consideran puntos de apoyo y esperanza. En el caso de los jóvenes EMTP, aun cuando el apoyo estratégico no sea explícito, el soporte emocional es un mecanismo de contención de la posible frustración que podría ocasionar no alcanzar las metas deseadas.

Soy el primer miembro de mi hogar que va estudiar la educación superior y se nota que mi mamá le enorgullece que su hijo. [sic] Con mi papá la relación de convivencia es excelente. Es una persona muy abierta que se cuida de no intervenir en mis asuntos y siempre me demuestra su apoyo. (Hombre, EMHC)

Mi madre y mi hermana mayor, que ya no vive con nosotras sino con su pareja, no fueron a la universidad, así que ellas y todos en general están súper orgullosas [sic] de que yo pueda hacerlo. En todo caso, eso no es una carga para mí, sino que muy por el contrario, lo siento como un aliciente que me motiva a esforzarme para que me vaya bien, y que me impide decepcionarlas. Tengo un tío igual, el hermano menor de mi mamá, que también entró a estudiar ahora. Él tiene 29 años, y como no tiene hijos, me ve como si fuera su hija, y siempre me está apoyando. (Mujer, EMTP) 
En los relatos también se pone en evidencia que las elecciones vocacionales están influidas por los soportes institucionales. En los jóvenes de la EMHC, por el hecho de encontrarse en un espacio escolar enfocado en la prosecución de estudios, se despliega un horizonte de posibilidades. Es un abanico amplio, del que tienden a elegir y seguir estudios centros de estudios de más alta valoración social.

Por contrapartida, si analizamos los soportes institucionales EMTP, varias de las narraciones de jóvenes de buen rendimiento académico dejan ver que la preparación recibida no estuvo orientada a la prosecución de estudios en la educación superior (especialmente en jóvenes EMTP), lo que constituirá un gran obstáculo para alcanzar las metas propuestas. Es más, la preparación escolar se representa como incompleta e insuficiente, y se expresa además que las expectativas de los agentes escolares respecto de las capacidades de progreso de estos jóvenes también es un obstáculo al cual se debe hacer frente. En este sentido, algunos de estos jóvenes despliegan estrategias académicas complementarias de alta exigencia a fin de suplir las diferencias académicas.

Con todo, cuando nos detenemos a mirar las narrativas, advertimos que los jóvenes de la EMTP expresan que cuentan con menores herramientas de acceso para concretar la aspiración de ingresar a la educación. Estos jóvenes manifiestan que disponen de menos recursos de información y beneficios que los ayuden a concretar sus metas. En este sentido, los soportes familiares, las instituciones de educación escolar y los dispositivos de apoyo institucionales emergen como factores limitantes.

Es que en sí los colegios no te pasan materia que entra en la PSU, como que en realidad del mercado está tan bien hecho para que nosotros paguemos el pre-u, que no nos pasan la materia. [...] Entonces, nosotros le pedimos a la profe que porfa lo viera porque en realidad no todos vamos a pre-u y queremos aprender igual. (Mujer EMTP)

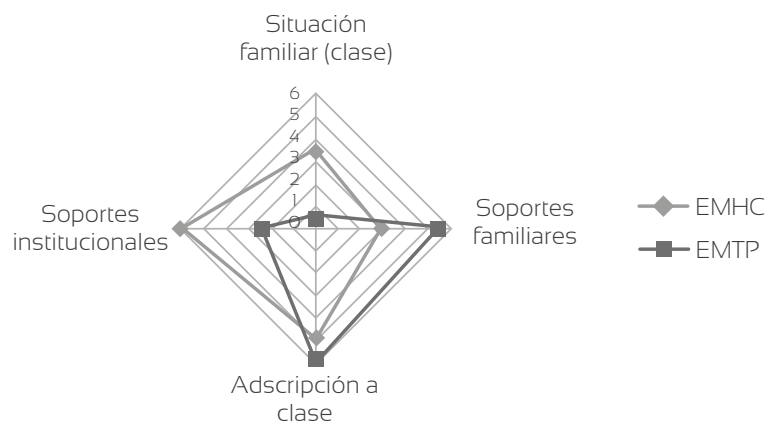

Figura 2. Dimensiones posición sociohistórica según modalidad de estudios 


\section{Eje voz}

Una de las principales constataciones de la investigación se refiere a la forma en que los jóvenes narran sus recorridos. De acuerdo con lo que se puede evidenciar, los relatos, si bien denotan experiencias constreñidas por los límites estructurales para los jóvenes de mayor vulnerabilidad económica y social, también manifiestan que ellos se muestran activos en la construcción de sus propias trayectorias y recorridos, autorrepresentándose como capaces de superar distintas barreras.

Un futuro auspicioso, con distintas alternativas posibles de alcanzar, es un elemento común en los relatos de los jóvenes. Este optimismo toma la forma de aspiraciones de mejoramiento de las condiciones de vida y realización personal para los estudiantes de sectores medios y medios bajos, aunque no necesariamente de movilidad social ascendente.

Los jóvenes afirman que se sienten sujetos activos y capaces de alcanzar sus metas y manejar las contingencias futuras, apelando a que el esfuerzo personal es la vía que asegura la consecución de futuro. El reconocimiento de las barreras estructurales se evidencia en algunos casos, pero nunca el logro de metas se verá absolutamente obstaculizado por los constreñimientos. Los sujetos asumen en sus imaginarios un rol activo en sus vidas, apelando al esfuerzo personal como un recurso al que pueden acudir en cualquier momento para tomar control de su porvenir.

El optimismo se forja, pues, en la creencia en un modelo meritocrático en el que es posible alcanzar las metas propuestas a través del esfuerzo. Los jóvenes conciben el esfuerzo personal como el conjunto de acciones que es necesario llevar a cabo para alcanzar los objetivos, sin que estas estén forzosamente asociadas a la consecución de buenos rendimientos y resultados académicos. Tal como se muestra en la comparación de casos, no existe relación entre las convicciones sobre los factores asociados al logro de metas y el reconocimiento del esfuerzo académico realizado en el pasado. En algunos casos, se evidencia una disonancia entre las metas alcanzadas, el esfuerzo desplegado y las aspiraciones futuras.

A mí me gusta harto salir, aunque tampoco lo hago tanto. De todos modos, creo que ese podría llegar a ser un problema más adelante, si es que una llegara a perder los estribos con eso. O sea, yo no lo hago mucho, pero creo que podría ser algo malo, y que podría atentar contra los planes que me he planteado. [...] En todo caso, tengo claro que para llegar ahí es súper importante el esfuerzo que haga ahora, y que de alguna forma, es también lo que me ha permitido llegar acá. Sin él, es muy difícil alcanzar los objetivos que uno se propone. (Mujer, EMTP)

Nunca me he planteado concienzudamente los pasos que voy a dar de acá al futuro porque prefiero no programar las cosas en exceso y dejar que fluyan por sí mismas, pero sí tengo claro que el logro de 
las metas personales depende exclusivamente de uno mismo: si uno quiere conseguir algo tiene que poner todo de su parte. (Hombre, EMHC)

Yo creo que a lo mejor me encuentre con una realidad que no es la que yo creo, entonces a lo mejor eso va a ser chocante, pero en sí estoy como bastante dispuesta a enfrentar cualquier tipo de realidad que me Ilegue a encontrar. (Mujer EMHC)

En este sentido, se puede hipotetizar que este reconocimiento discursivo al esfuerzo se funda en imaginarios sociales hegemónicos transmitidos (en la familia, la escuela y los medios de comunicación), en los cuales si bien se comunica el valor del mérito en el logro de objetivos, los sentimientos de injusticia y frustración frente a las desigualdades que se hacen latentes en las experiencias biográficas familiares chilenas provocan que el esfuerzo, en algunos casos, se circunscriba a una dimensión discursiva-proyectiva. El lugar central que ocupa el esfuerzo, fundado y transmitido por imaginarios hegemónicos como las familias, los centros educacionales y los medios de comunicación, es potenciado por las desfavorables situaciones socioeconómicas que experimentan muchos hogares que ven en él la única salida (Araújo y Martucelli, 2015). Es más, el optimismo y el valor asignado a los principios que fundan el modelo meritocrático basado en el esfuerzo personal activan el sentimiento de autonomía y control sobre la vida, como observan Rudd y Evans (1998). Estos jóvenes, si bien tienden a validar el mérito personal como mecanismo de asignación de posición en la estructura, reconocen que en su perspectiva de futuro tendrán que seguir batallando con el fin de acceder a mejores condiciones de vida que las actuales, en un sistema que comprenden que tiene fisuras, pero que para ellos se pueden solucionar.

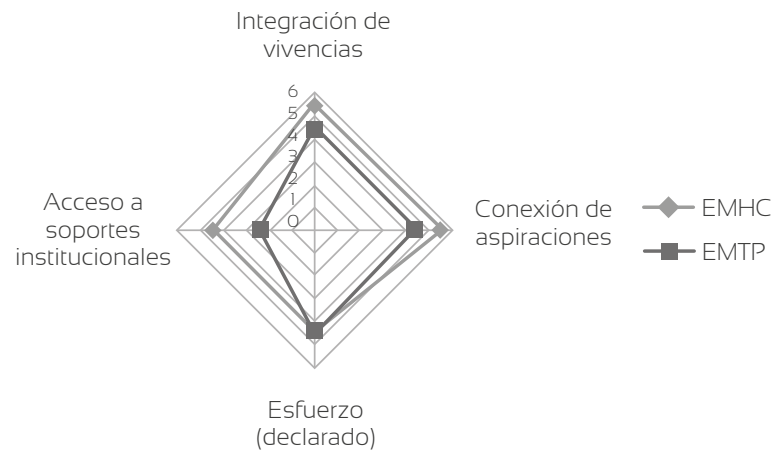

Figura 3. Dimensiones de la voz según tipo de recorrido. 


\section{Conclusiones}

Mediante el análisis de las subjetividades y de las narrativas se puede comprender el marco simbólico en el que se da la configuración de las aspiraciones y expectativas juveniles, y conocer cómo se vive la singularidad de las experiencias previas al egreso de la educación secundaria. Constatamos que la educación superior es una aspiración abierta a jóvenes con distintos intereses y distintas trayectorias escolares y socioculturales. El ingreso a la educación superior se percibe como la posibilidad de configurar un proyecto de vida que conjuga el desarrollo profesional con los intereses personales. Para los jóvenes de la educación técnica adquiere un valor instrumental, puesto que constituye un medio para alcanzar cualificación profesional, garantizar inserción y estabilidad laboral. Para los jóvenes de la educación científica o humanista se asocia a un sentido social o individual, a la búsqueda de un proyecto con sentido.

No obstante estas diferencias, para todos los jóvenes el escenario futuro se proyecta como auspicioso, un optimismo que se forja, pues, en la creencia de que es posible alcanzar las metas propuestas a través del esfuerzo. Los jóvenes conciben el esfuerzo personal como el conjunto de acciones que es necesario llevar a cabo para alcanzar los objetivos, sin que estas acciones estén forzosamente asociadas a la consecución de buenos rendimientos y resultados académicos. Se puede hipotetizar que este reconocimiento discursivo al esfuerzo se funda en imaginarios sociales hegemónicos transmitidos (entiéndase por esto distintas instancias de socialización), en los cuales el valor del mérito en el logro de objetivos se circunscribe a una dimensión asociada a la capacidad de navegar, sortear barreras (ser un híper-actor [Araujo y Martucelli, 2011]), más que a las propias lógicas meritocráticas de los sistemas y modelos educativos basados en pautas de demostración de talentos y conocimientos.

El predominio de estos sentidos, que se fundan en el optimismo y el valor asignado al modelo meritocrático, activa en los jóvenes el sentimiento de autonomía y control sobre la vida. Los jóvenes tienden a retratar una experiencia que está constreñida por los límites estructurales, con marcos de comprensión y significación en los que aparecen como sujetos activos en la construcción de sus propias trayectorias y recorridos, sintiéndose a su vez, en cierta medida, responsables de los constreñimientos estructurales. Asociado a lo anterior, el sentimiento de control sobre el futuro, mediado por una supuesta activación del esfuerzo personal, es uno de los elementos que refuerza la prolongada estadía en el sistema educativo y el llamativo optimismo de los jóvenes.

Al reconocer la ambivalencia de agencia, control y estructura implicada en el proceso, también podemos señalar que el análisis por eje muestra que los procesos de construcción identitaria profesional implicados

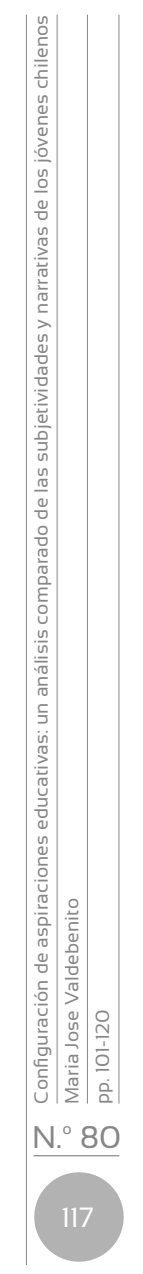


en los procesos formativos de nivel secundario cobran trascendencia. Las vivencias educativas y laborales que ofrece la educación técnica pareciesen apoyar el desarrollo de un escenario que favorece una forma distintiva de tránsito a la adultez y a la definición de rutas alternativas para alcanzar los propósitos individuales. Se configura un marco en el que los jóvenes, al tiempo que buscan su vocación, tratan de diseñar estrategias para sortear las condicionantes estructurales y reconocer oportunidades futuras. Asumiendo que deben aprender a moverse en escenarios cada vez más inciertos, desarrollan ciertas capacidades que les permitirán reconstruir itinerarios de manera permanente, por ejemplo, a través de la alternancia de educación y trabajo.

Más allá de estas potencialidades, se debe reseñar que si bien las instituciones escolares de carácter técnico ofrecen oportunidades de aprendizaje favorables en el sentido descrito, también tenderían a reducir ciertas oportunidades. Su énfasis orientado exclusivamente a la formación para el trabajo limitaría las posibilidades de imaginar y pensar diversas alternativas futuras y de contar con capacidades que permitan sortear ciertas barreras de selectividad institucional. Junto a ello, los procesos de apoyo vocacional son limitados, lo que da lugar al desafío de repensar su modelo formativo. Podría llegar a cuestionarse las capacidades institucionales para la entrega de herramientas, conocimientos y habilidades que permitan a los jóvenes alcanzar sus metas y objetivos y sortear los escollos que presentan las trayectorias posteriores al egreso, cada vez más complejas. Todo este escenario hace que la escuela técnica, entendida como una institución social destinada a dar soporte a los jóvenes en esta etapa, se vea ciertamente interpelada.

\section{Referencias}

Araujo, K. y Martuccelli, D. (2012). Desafíos comunes: retrato de la sociedad chilena y sus individuos, tomos I y 1 . LOM.

Arfuch, L. (2007). El espacio biográfico: dilemas de la subjetividad contemporánea. Fondo de Cultura Económica.

Atkins, L. (2017). The odyssey: School to work transitions, serendipity and position in the field. British Journal of Sociology of Education, 38(5), 641-655.

Ball, S., Davies, J., David, M. y Reay, D. (2002). 'Classification' and 'Judgement': Social class and the 'cognitive structures' of choice of Higher Education. British Journal of Sociology of Education, 23(1), 51-72.

Ball, S.; Maguire, M. y Macrae, S. (2000). Choice, pathways, and transitions post-16: New youth, new economies in the global city. Routledge/ Falmer. 
Bennett, R. J., Glennester, H. y Nevison, D. (1992). Learning Should Pay. BP Educational Service.

Bertaux, D. (1982). Stories as Clues to Sociological Understanding. En P. Thompson (ed.): Our Common History: The Transformation of Europe. Pluto Press.

Cabrera, A. y La Nasa, S. (2002). Understanding the college-choice process. New Directions for Institutional Research, 107, 5-22.

Canales, M., Opazo, A. y Camps, J. (2016). Salir del cuarto. Expectativas juveniles en el Chile de hoy. Última Década, 24(44), 73-108.

Centro de Investigación y Desarrollo de la Educación (2010). Encuesta a actores del sistema educacional. http://www.cide.cl/documentos/ Informe_IX_Encuesta_CIDE_2012.pdf

Clarke, J., Hall, S., Jefferson, T. y Roberts, B. (1981). Subcultures, cultures and class. En T. Bennet, G. Martin, C. Mercer y J. Wallacott (eds.). Culture, Ideology and Social Process. Batsford.

Dale, R., Kasepov, Y., Rinne, R. y Robertson, S. (2016). Scales, Discourses and Institutions in the Governance of Educational Trajectories in Europe. En Walther, Parreira, Cuconato y Rogres (eds.). Governance of Educational Trajectories in Europe: Pathways, Policy and Practice. Blommsbury.

Dubet, F. (2005). Los estudiantes. CPU-e Revista de Investigación Educativa, 1, 1-78.

Dubet, f. (2011). Repensar la justicia social. Contra el mito de la igualdad de oportunidades. Siglo xxı.

Elder, G. y Giele, J. (2009). Life-course studies: An evolving Field. En G. Elder y J. Giele (Eds.), The craft of life-course research (pp. 1-25). The Guilford Press.

Elias, N (1997 [1939]). La sociètè des individus. Agora Dèbats.

Furlong, A. \& Cartmel, F. (1997). Young People and Social Change: Individualization and Risk in Late Modernity. Series: Sociology and Social Change. Open University Press: Buckingham, England.

Gaete, T. \& Soto, A. (2012). Esta es mi trayectoria, este es mi trabajo: narrativas e identidad en el trabajo en Chile. Psykhe, 21(2), 47-59.

Gibbons, S. y Vignoles, A. (2009). Access, Choice and Participation in Higher Education. Centre for Education Economics London School Of Economics and Political Sciences Draft Papers, 101(1), 1-52.

Grytnes, R. (2011). Making the right choice! Inquiries into the reasoning behind young people's decisions about education. Young, 19, 333-351.

Hart, C. (2013). Aspirations, Educations and Social Justice. 
Hatcher, R. (1998). Class Differentiation in Education: rational choices?, British Journal of Sociology of Education, 19(1), 5-24. https://doi. org/10.1080/0142569980190101

Heinz, W. y Krüger, H. (2001). Life course: innovations and challenges for social research. Current Sociology, 49(2), 29-45. SAGE Publications.

Hodkinson, P. y Sparkes, A. C. (2006). Careership: A sociological theory of career decision making. British Journal of Sociology of Education, 18(1), 29-44.

Jacinto, C., (2010). La construcción social de trayectorias laborales de jóvenes: políticas, instituciones, dispositivos y subjetividades. Teseo.

Larrañaga, O., Cabezas, G. y Dusaillant, F. (2013). Educación técnico profesional: trayectoria educacional e inserción laboral de una cohorte de alumnos. PNUD. http://www.pnud.cl/areas/ReduccionPobreza/2013/ pdf_EMTP/Estudio_EMTP_PNUD.pdf

Lehmann, W. (2004). For some reason, I get a little scared': structure, agency, and risk in school-work transitions. Journal of Youth Studies, 7(4), 379-396.

PNUD (2017). Orígenes, cambios y desafíos de la desigualdad en Chile. UNDP.

Rivera, L. (2012). Hiring as cultural matching: The case of elite professional service firms. American Sociologial Review, 77(6), 999-1022.

Roberts, K. (1968). The entry into employment: An approach towards a general theory. The Sociological Review, 16, 2, 165-184.

Roberts, K. (1975). The development theory of occupational choice: A critique and an alternative. En G. Esland, G. Salomon y M. Speakman (eds.), People and Work. Open University Press.

Rudd, P.W. y Evans, K. (1998) Structure and Agency in Youth Transitions: Student Experiences of Vocational Further Education. Journal of Youth Studies, 1(1), 39-62. https://doi.org/10.1080/13676261.1998.105 92994

Sellar, S., Gale, T. y Parker, S. (2011). Appreciating aspirations in Australian higher education. Cambridge Journal of Education, 41(1), 37-52.

Sepúlveda, L. y Valdebenito, M. J. (2018). Educational-work projects and postgraduate pathways of secondary students in Chile: Individual strategies in an unequal education system. En S. Carney y M. Schweisfurth (eds.) Equity in and through education: Changing contexts, consequences, and contestations. Brill-Sense Publishers.

Torres, R. y Zenteno, M.L. (2011). El sistema de educación superior: Una mirada desde las instituciones y sus características. En M. Jiménez, y F. Lagos, (ed.), Nueva geografía de la educación superior y de los estudiantes: Una cartografía del sistema chileno, su actual alumnado y sus principales tendencias. Ediciones Universidad San Sebastián. 